\title{
RIESGO CARDIOVASCULAR, OCUPACIÓN Y RIESGOS LABORALES EN UNA POBLACIÓN LABORAL DE CATALUNYA
}

\author{
JOSEP MARÍA MOLINA ARAGONÉS \\ Médico del Trabajo y Técnico Superior de Prevención de Riesgos Laborales del \\ Sistema Emergències Mèdiques (SEM SA). \\ L'Hospitalet de Llobregat. \\ Barcelona
}

\section{RESUMEN}

Objetivo: Determinar la prevalencia de factores de riesgo cardiovascular en población trabajadora de Catalunya, analizar su relación con la actividad laboral y los riesgos laborales asociados a esta.

Material y métodos: Se analizaron datos de factores de riesgo cardiovascular (tabaquismo, hipertensión, dislipemia y diabetes) durante el periodo de Enero a Junio de 2.006 en el ámbito territorial de Catalunya, y de los riesgos laborales obtenidos a partir de las evaluaciones de riesgos y de la actividad profesional y empresarial de los trabajadores incluidos en el estudio.

Resultados: Se obtuvieron datos de 2.573 trabajadores, $1.766(68,7 \%)$ hombres y $806(31,3 \%)$ mujeres. $2.233(86,9 \%)$ presentaron un riesgo cardiovascular bajo, $304(11,8 \%)$ un riesgo cardiovascular ligero y $34(1,3 \%)$ un riesgo cardiovascular moderado-alto. Se evidenciaron diferencias significativas en el comportamiento del riesgo cardiovascular en función de dicha actividad laboral $(\mathrm{p}<0,00)$.

Conclusiones: La distribución de los factores de riesgo cardiovascular no fue homogénea en las diferentes actividades laborales. Distintos sectores de actividad llevan implícitos hábitos y conductas, que son las que definitivamente influyen en el riesgo cardiovascular. Este se encuentra asociado a factores de tipo individual y no tanto a las condiciones laborales. El abordaje preventivo deberá elaborarse en función de los diferentes sectores de actividad.

\section{PALABRAS CLAVES}

Factores de riesgo, Medicina laboral, Enfermedades cardiovasculares.

\section{ABSTRACT}

Objective: Determining the prevalence of cardiovascular risk factors in Catalan workers; analysing their relationships with labour activity and the labour risks associated with this.

Materials and Methods: Cardiovascular risk factors data (nicotine poisoning, hypertension, dyslipemia, and diabetes) from January to June of 2006 in Catalonia, and labour risks data obtained from evaluations of professional and business activity of workers object of this study were analysed.

Results: Data from 2.573 workers $\left(1.766\left(68^{\prime} 7\right.\right.$ $\%)$ men and 806 (31'3\%) women) were obtained. $2.233\left(86^{\prime} 9 \%\right)$ present a low cardiovascular risk, 304 (11'8) present a light cardiovascular risk, and 34 (1'3 \%) a high-medium cardiovascular risk. Significant differences in the cardiovascular risk behaviour according to this labour activity were shown $(\mathrm{p}<0,00)$.

Conclusions: The distribution of cardiovascular risk factors was not homogeneous in the different labour activities. Different activity sectors involve habits and behaviours which definitively influence cardiovascular risk. You can find this risk associated to factors of individual type and not to labour conditions. The preventive treatment must be prepared according to different activity sectors.

\section{KEY WORDS}

Risk factors, Occupational Medicine, Cardiovascular diseases. 


\section{INTRODUCCIÓN}

Las enfermedades del aparato cardiocirculatorio son la primera causa de morbi-mortalidad en Catalunya y el segundo motivo de consulta en atención primaria $^{1}$.

Respecto a los factores de riesgo a estas asociados, se describe un patrón epidemiológico multifactorial, con factores que en muchas ocasiones se presentan asociados y entre los que sen encuentran el consumo de tabaco, la Hipertensión arterial (HTA), la hipercolesterolemia (HC), la diabetes y la obesidad entre otros.

Del mismo modo, otros riesgos potenciales de la enfermedad cardiovascular incluyen variables socioeconomicas entre las que se encuentran condiciones medioambientales y laborales ${ }^{2,3}$.

No existen dudas sobre el problema de salud pública que suponen este tipo de enfermedades en nuestro medio y de la necesidad de realizar intervenciones para disminuir su incidencia. La actuación sobre factores modificables es una estrategia de prevención primaria de la que disponemos de evidencia científica epidemiológica y clínica que avala su efectividad ${ }^{1}$.

El objetivo principal de este estudio es determinar la prevalencia de factores de riesgo cardiovascular en una población trabajadora de Catalunya y analizar su relación con la actividad laboral y los riesgos laborales asociados a esta.

\section{MATERIAL Y MÉTODO}

Se analizaron los datos de los factores de riesgo cardiovascular obtenidos en el momento de la práctica de exámenes de salud preventivos durante el periodo de Enero a Junio de 2.006 en el ámbito territorial de Catalunya. Se estimó un tamaño muestral necesario de 2402 trabajadores, para obtener una determinación de prevalencia $\mathrm{p}=\mathrm{q}=0,5$, con un intervalo de confianza del $95 \%$ y un margen de error del 2\%. La inclusión de los trabajadores se efectuó de manera simple, mediante la asignación temporal en el periodo establecido.

A efectos de clasificación se consideró fumador aquel individuo que manifestó el consumo regular de tabaco en cualquiera de sus formas (tabaco, ciga- rros $\mathrm{u}$ otros) en el momento del reconocimiento o bien que manifestaba haber abandonado el hábito en los doce meses previos al mismo.

Se considero afecto de hipercolesterolemia (HC) a aquellos trabajadores con Colesterol plasmático $\geq 200 \mathrm{mg}$. / $100 \mathrm{ml}$. y aquellos que manifestaron dicha enfermedad como antecedente y efectuaban tratamiento dietético o farmacológico.

Respecto a la Hipertensión arterial (HTA) se consideraron casos aquellos individuos con cifras de TAS (Tensión arterial sistólica) $\geq 140 \mathrm{mmHg}$, TAD (Tensión arterial diastólica) $\geq 90 \mathrm{mmHg}$ en el momento del reconocimiento médico o bien aquellos casos que manifestaron padecer HTA y/o recibían tratamiento anti-hipertensivo.

Respecto a la diabetes, se incluyeron aquellos casos con cifras de glicemia basal $\geq 110 \mathrm{mg} \%$ o bien manifestaron padecer dicha enfermedad o encontrarse en tratamiento mediante antidiabéticos orales (ADO) o insulina.

El riesgo coronario se calculó utilizando las tablas de riesgo coronario calibradas para la población española de Marrugat et $\mathrm{al}^{4}$. Estas tablas de riesgo cardiovascular intentan adaptar las predicciones al contexto del estado español y países del sur de Europa. La estimación que ofrecen del riesgo coronario es más cercana al riesgo observado en nuestro medio que la sobreestimación efectuada por las tablas de Framingham originales habitualmente utilizadas. En su elaboración se tienen en cuenta edad y sexo, HTA, $\mathrm{HC}$, diabetes y tabaquismo, variables incluidas en el análisis. Los menores de 35 años fueron incluidos sistemáticamente en el primer grupo de edad.

Los riesgos laborales se recogieron, de forma cualitativa, a partir de los datos reflejados en las evaluaciones de riesgo de los puestos de trabajo de los individuos que se incluyeron en el estudio.

Para facilitar el análisis de los datos, la actividad laboral se clasificó en cuatro categorías: administración, servicios, industria y construcción. Estas categorías fueron atribuidas por el propio autor en función de las características de la evaluación de riesgos y los datos recogidos en la anamnesis clínica. En el grupo de administración se incluyeron aquellos trabajadores cuya actividad consiste princi- 
palmente en tareas administrativas mediante utilización de pantallas de visualización de datos o análogas. En la categoría de servicios, que se diferenció de la de administración, se incluyeron trabajadores de limpieza, chóferes, personal sanitario y docente, personal en relación directa con clientes y otros superponibles. En la categoría industrial se incluyeron aquellos operarios y montadores industriales del tipo de torneros, fresadores, trabajadores en líneas de montajes, etc. Por último, en la categoría de construcción se incluyeron aquellos cuya tarea principal es la construcción en si misma o bien trabajos auxiliares que se desarrollan principalmente en obras de construcción. Los trabajadores fueron clasificados individualmente, y en caso de duda en la asignación, su inclusión se resolvía mediante la ayuda de un colaborador externo con formación en Medicina del Trabajo.

El tratamiento de los datos se ha efectuado mediante análisis univariante presentando los datos estratificados por actividad laboral. El análisis biva- riante se ha efectuado mediante test de chi-cuadrado en el caso de ambas variables discretas y " $t$ " de Student para la comparación de variables continuas. Para el estudio de la variable edad, de carácter continuo, se ha utilizado el test de ANOVA cuando ha sido necesario. El análisis multivariante se ha efectuado calculando la Odds de Prevalencia (POR) mediante técnica de regresión logística. La variable dependiente utilizada ha sido el riesgo cardiovascular, obtenido mediante las tablas de Marrugat, el cual se ha dicotomizado en riesgo cardiovascular bajo y riesgo cardiovascular ligero-moderado.

El tratamiento de los datos se ha efectuado mediante el paquete estadístico SPSS v 8.0. El nivel de significación admitido ha sido $\mathrm{p}=.05$.

\section{RESULTADOS}

Se analizaron los datos de 2.573 trabajadores, de los cuales $1.766(68,7 \%)$ fueron hombres y 806 $(31,3 \%)$ mujeres. La tabla 1 muestra la estadística

Tabla 1. Distribución de las variables incluidas en el estudio, estratificadas por ocupación.

\begin{tabular}{|c|c|c|c|c|c|c|c|}
\hline & & \multicolumn{6}{|c|}{ Actividad laboral } \\
\hline & & Administración & Servicios & Industria & Construcción & Total & \\
\hline & & $\mathrm{N}(\%)$ & $\mathrm{N}(\%)$ & $\mathrm{N}(\%)$ & $\mathrm{N}(\%)$ & $\mathrm{N}(\%)$ & $p$ \\
\hline \multirow[t]{2}{*}{ Sexo } & Hombre & $290(52,6 \%)$ & $495(55,6 \%)$ & $651(81,8 \%)$ & $330(98,5 \%)$ & $1766(68,7 \%)$ & $<0,01^{a}$ \\
\hline & Mujer & $261(47,4 \%)$ & $395(44,4 \%)$ & $145(18,2 \%)$ & $5(1,5 \%)$ & $806(31,3 \%)$ & \\
\hline \multirow[t]{2}{*}{ Tabaco } & sl & $168(30,5 \%)$ & $297(33,4 \%)$ & $378(47,5 \%)$ & $152(45,4 \%)$ & $995(38,7 \%)$ & $<0,01^{a}$ \\
\hline & NO & $383(69,5 \%)$ & $593(66,6 \%)$ & $418(52,5 \%)$ & $183(54,6 \%)$ & $1577(61,3 \%)$ & \\
\hline \multirow[t]{2}{*}{ HTA } & SI & $85(15,4 \%)$ & $163(18,3 \%)$ & $184(23,1 \%)$ & $81(24,2 \%)$ & $513(19,9 \%)$ & $0,03^{a}$ \\
\hline & NO & $466(84,7 \%)$ & $727(81,7 \%)$ & $612(76,9 \%)$ & $254(75,8 \%)$ & $2059(80,1 \%)$ & \\
\hline \multirow{2}{*}{$\mathrm{HC}$} & SI & $227(44,3 \%)$ & $435(48,9 \%)$ & $383(48,1 \%)$ & $166(49,6 \%)$ & $1261(49,0 \%)$ & $0,08^{\mathrm{a}}$ \\
\hline & NO & $307(55,7 \%)$ & $455(51,1 \%)$ & $413(51,9 \%)$ & $169(50,4 \%)$ & $1311(51,0 \%)$ & \\
\hline \multirow[t]{2}{*}{ Diabetes } & SI & $14(2,5 \%)$ & $21(2,4 \%)$ & $34(4,3 \%)$ & $25(7,5 \%)$ & $94(3,7 \%)$ & $<0,01^{a}$ \\
\hline & NO & $537(97,5 \%)$ & $869(97,6 \%)$ & $762(95,7 \%)$ & $310(92,5 \%)$ & $2478(96,3 \%)$ & \\
\hline Edad $^{*}$ & & $37,6(9,6)$ & $37,8(9,8)$ & $38,2(11,4)$ & $38,4(12,2)$ & $38,0(10,5)$ & $0,61^{b}$ \\
\hline Total & & $551(21,4 \%)$ & $890(34,6 \%)$ & $796(30,9 \%)$ & $335(13,0 \%)$ & \multicolumn{2}{|c|}{$2573(100,0 \%)$} \\
\hline \multicolumn{8}{|c|}{$\begin{array}{l}\text { Tabaco: Consumo de tabaco en cualquiera de sus formas o exfumador de menos de doce meses. } \\
\text { HTA: Hipertensión arterial. TAS } \geq 140 \text { o TAD } \geq 90 \text { o tratamiento anti-hipertensor. } \\
\text { HC: Hipercolesterolemia. Colesterol }>200 \mathrm{mg} \% \text { o HC conocida o tratamiento hipolipemiante } \\
\text { Diabetes: Glicemia }>100 \mathrm{mg} \% \text { o diabetes conocida o tratamiento antidiabético (ADO o insulina) } \\
{ }^{*} \text { Edad: Expresada como [Media (Desviación estándar)] } \\
\text { a: ji-cuadrado } \\
\text { b: ANOVA }\end{array}$} \\
\hline
\end{tabular}


Tabla 2. Distribución del riesgo cardiovascular (RCV)*, en función de la actividad laboral. Actividad laboral

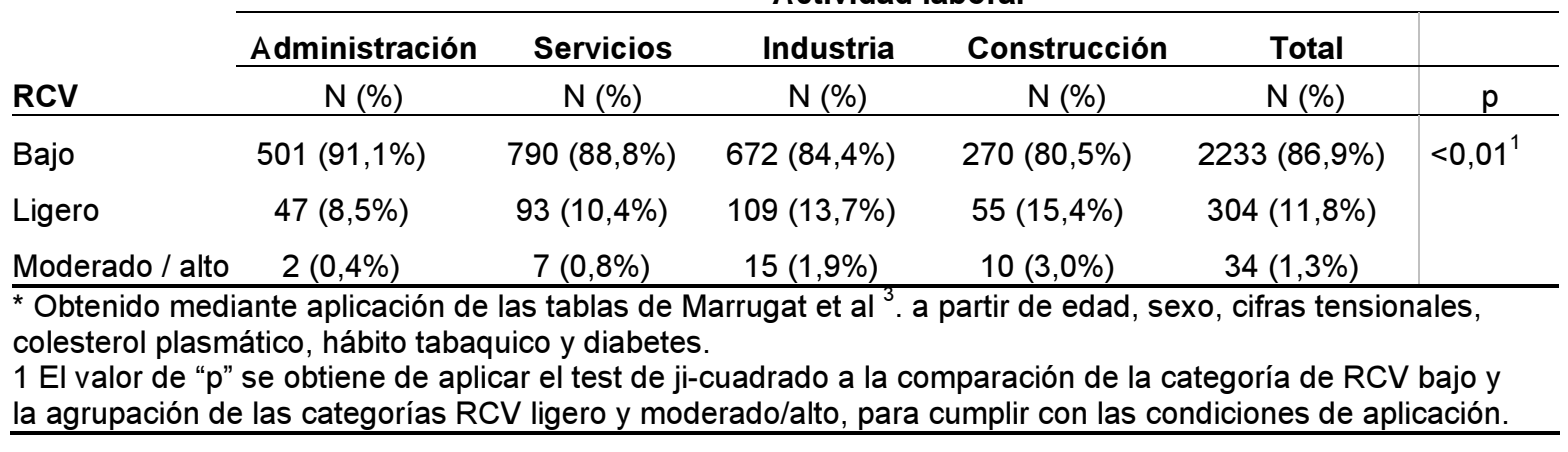

descriptiva estratificada por ocupación. La distribución de la variable sexo en función de la actividad laboral no resultó homogénea, detectándose diferencias significativas en la misma $(\mathrm{p}<0,00)$.

La edad media y la desviación estándar (DE) de la muestra fue de 37,98 $\pm 10,49$ años. Por sexos, la edad media y DE fueron de $39,0 \pm 10,8$ y $35,7 \pm 9,4$ para hombres y mujeres respectivamente $(\mathrm{p}<0,00)$. La distribución según la actividad laboral se muestra en la tabla 1. No se evidenciaron diferencias estadísticamente significativas en la distribución de la variable en función de la edad $(p=0,60)$.

Respecto a los factores de riesgo cardiovascular, el $19,9 \%$ de los individuos presentaron criterios de HTA. 426 hombres $(24,1 \%)$ y $87(10,8 \%)$ mujeres cumplían estos criterios. Estos porcentajes mostraron diferencias estadísticamente significativas entre ambos sexos $(\mathrm{p}<0,00)$. La distribución de la HTA en función de la actividad laboral se muestra en la tabla 1. También se evidenciaron diferencias estadísticamente significativas en la proporción de hipertensos en función de la actividad laboral $(\mathrm{p}<$ $0,00)$.

Respecto al consumo de tabaco, 995 individuos $(38,7 \%)$ declararon consumo regular en cualquiera de sus formas. De estos, 716 (40,5\%) eran hombres y $279(34,6 \%)$ mujeres. Se evidenciaron diferencias estadísticamente significativas en el consumo de tabaco respecto al sexo $(\mathrm{p}<0,00)$. La distribución en función de la actividad laboral se muestra en la tabla 1. También se evidenciaron diferencias estadísticamente significativas en el consumo de tabaco en función de la actividad laboral $(\mathrm{p}<0,00)$.
94 individuos $(3,7 \%)$ cumplían criterios de diabetes. 85 de ellos $(4,8 \%)$ eran varones y $9(1,1 \%)$ mujeres. Se detectaron diferencias estadísticamente significativas en la prevalencia de la diabetes en función del sexo $(p<0,00)$. La distribución en función de la actividad laboral se muestra en la tabla 1. También se evidenciaron diferencias en la prevalencia de diabetes en función de la actividad laboral $(p<0,00)$.

La figura 1 muestra la distribución de los factores de riesgo cardiovascular considerados en función de las actividades laborales.

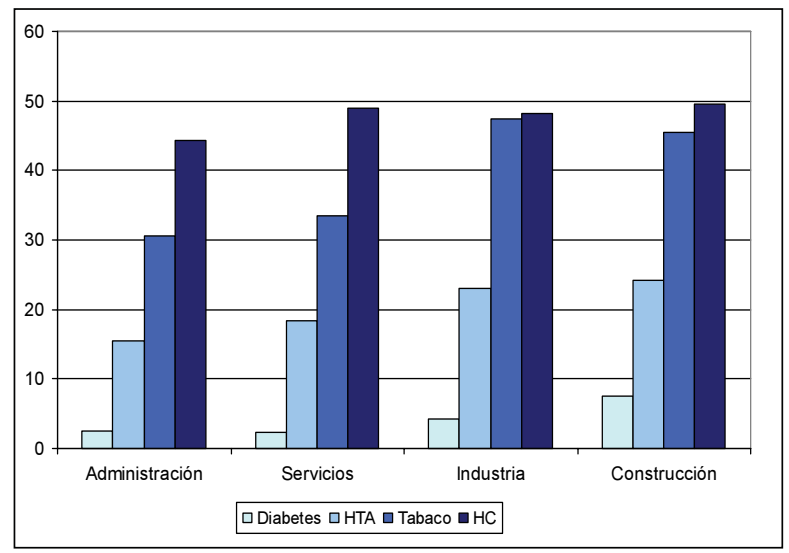

Figura 1. Distribución de los factores de riesgo cardiovascular (\%) en función de la actividad laboral (HTA: Hipertensión arterial; HC: Hipercolesterolemia).

Respecto al riesgo cardiovascular, 2233 individuos $(86,9 \%)$ presentaron un riesgo cardiovascular bajo, $304(11,8 \%)$ un riesgo cardiovascular ligero y 
$34(1,3 \%)$ un riesgo cardiovascular moderado-alto. Por sexos, el riesgo cardiovascular fue ligero-moderado en $298(16,9 \%)$ hombres y $40(5,0 \%)$ mujeres. Esta diferencia resultó estadísticamente significativa $(\mathrm{p}<0,00)$ de modo que debemos admitir que el comportamiento del riesgo cardiovascular es diferente en función del sexo. La distribución del riesgo cardiovascular en función de la actividad laboral se muestra en la tabla 3. (Para ambos análisis se unieron las categorías de riesgo ligero y moderado). También se evidenciaron diferencias significativas en el comportamiento del riesgo cardiovascular en función de dicha actividad laboral $(\mathrm{p}<0,00)$.

La exposición a los diferentes riesgos laborales se muestra en la tabla 4. Destacan por su prevalencia las pantallas de visualización de datos $(46,5 \%)$, la manipulación manual de cargas $(37,0 \%)$, el ruido $(27,3 \%)$ y la exposición respiratoria a humos, polvos $\mathrm{y} / \mathrm{o}$ vapores $(22,7 \%)$.

Los resultados del análisis multivariante se muestran en las tablas 4 y 5. Destaca una relación significativa, tras ajustar por edad, sexo y ocupación, entre el riesgo derivado de la adopción de posturas forzadas y el riesgo cardiovascular.

\section{DISCUSIÓN}

La distribución de los factores de riesgo cardiovascular no es homogénea en las diferentes activida- des laborales. El riesgo asociado a la misma sigue una progresión ascendente desde las tareas de tipo administrativo hasta las ocupaciones relacionadas con la construcción, lo que intuitivamente asociamos a trabajos con complejidad ascendente, mayores exigencias físicas y mayor número de riesgos asociados. La asociación del RCV con la carga física de trabajo ha sido considerada por algunos autores ${ }^{14}$.

La prevalencia de factores de RCV es mayor, y significativamente distinta en muchos casos, en sectores como la industria y la construcción, situación que ya han acreditado otros estudios previos ${ }^{5,6,7}$. El análisis parece constatar que, a pesar de los diferentes riesgos laborales, los sectores de actividad llevan implícitos unos hábitos y conductas higiénicas, que son las que definitivamente influyen en este RCV. En definitiva, este se encuentra asociado a factores de tipo individual y no tanto a las condiciones laborales, de modo que posiblemente deban elaborarse diferentes estrategias de abordaje en función de los diferentes sectores de actividad.

El estudio aporta la novedad de incluir riesgos laborales obtenidos a partir de las evaluaciones de los mismos, de los que no se han encontrado referencias en la bibliografía inicialmente consultada, salvo estudios diseñados a tal efecto con la consideración de algún único factor ${ }^{2,8}$.

Tabla 3. Asociación entre riesgo cardiovascular (RCV) y actividad laboral. Regresión logística ( RCV bajo vs. RCV ligero-moderado).

\begin{tabular}{|c|c|c|c|c|c|c|}
\hline \multirow[b]{2}{*}{ Actividades } & \multirow[b]{2}{*}{ POR (IC 95\%) } & \multirow[b]{2}{*}{$p$} & \multicolumn{2}{|l|}{ ES } & \multicolumn{2}{|l|}{ FRCV } \\
\hline & & & POR (IC 95\%) & $p$ & POR (IC 95\%) & $p$ \\
\hline Administración (1) / Servicios & $1,3(0,9-1,8)$ & 0,16 & $1,4(0,9-2,1)$ & 0,15 & $1,1(0,6-1,9)$ & 0,68 \\
\hline dministración (1) / Industria & $8(1,3-2,6)$ & $<0,01$ & $1,5(0,9-2,3)$ & 0,08 & $1,1(0,6-2,0)$ & 0,63 \\
\hline Administración (1) / Construcción & $2,4(1,6-3,6)$ & $<0,01$ & $1,6(0,9-2,7)$ & 0,10 & $1,3(0,7-2,6)$ & 0,38 \\
\hline Servicios (1) / Industria & $1,4(1,1-1,9)$ & $<0,01$ & $1,1(0,7-1,5)$ & 0,75 & $-1,6)$ & 0,93 \\
\hline Servicios (1) / Construcción & $1,9(1,3-2,7)$ & $<0,01$ & $1,1(0,7-1,8)$ & 0,62 & $1,2(0,6-2,2)$ & 0,60 \\
\hline Industria (1) / Construcción & $1,3(0,9-1,8)$ & 0,11 & $1,1(0,6-1,7)$ & 0,81 & $1,2(0,7-2,2)$ & 0,55 \\
\hline \multicolumn{7}{|c|}{$\begin{array}{l}\text { (1) Categoría de referencia } \\
\text { ES: Datos ajustados por edad y sexo } \\
\text { FCRV: Datos ajustados por edad, sexo y factores de riesgo cardiovascular (Consumo de tabaco, HC, HTA y } \\
\text { diabetes) } \\
\text { POR: Odds ratio de Prevalencia } \\
\text { IC } 95 \% \text { : Intervalo de confianza al } 95 \%\end{array}$} \\
\hline
\end{tabular}


Tabla 4. Prevalencia de riesgos laborales y asociación de estos con el riesgo cardiovascular (RCV). Estimación mediante ajuste de modelo de regresión logística ( RCV bajo vs. RCV ligero-moderado).

\begin{tabular}{lcccccccc}
\hline & \multicolumn{7}{c}{ ES } & \multicolumn{3}{c}{ Trabajo } & \\
\cline { 2 - 9 } Riesgos laborales & $\mathrm{N}(\%)$ & $\mathrm{POR}(\mathrm{IC} 95 \%)$ & $\mathrm{p}$ & $\mathrm{POR}(\mathrm{IC} 95 \%)$ & $\mathrm{p}$ & $\mathrm{POR}(\mathrm{IC} 95 \%)$ & $\mathrm{p}$ \\
\hline Ruido & $702(27,3 \%)$ & $1,8(1,4-2,3)$ & $<0,01$ & $1,2(0,8-1,6)$ & 0,29 & $1,0(0,7-1,6)$ & 0,83 \\
PVD & $1196(46,5 \%)$ & $0,5(0,4-0,6)$ & $<0,01$ & $0,8(0,6-1,1)$ & 0,18 & $1,0(0,6-1,7)$ & 0,97 \\
Humos, polvos & $585(22,7 \%)$ & $1,3(1,0-1,7)$ & 0,02 & $1,0(0,7-1,4)$ & 0,99 & $0,8(0,5-1,3)$ & 0,41 \\
MMC & $951(37,0 \%)$ & $1,5(1,2-1,9)$ & $<0,01$ & $1,2(0,9-1,6)$ & 0,26 & $1,0(0,7-1,5)$ & 0,83 \\
Turnicidad & $58(2,3 \%)$ & $1,9(1,0-3,6)$ & 0,03 & $2,0(0,7-5,0)$ & 0,15 & $1,8(0,7-4,7)$ & 0,21 \\
Agentes químicos & $52(2,0 \%)$ & $2,3(1,2-4,3)$ & 0,01 & $2,1(0,8-5,9)$ & 0,14 & $2,0(0,7-5,5)$ & 0,19 \\
Conducción & $338(13,1 \%)$ & $1,3(0,9-1,7)$ & 0,14 & $1,1,(0,7-1,6)$ & 0,80 & $1,0(0,6-1,6)$ & 0,97 \\
Movimientos repetitivos & $80(3,1 \%)$ & $0,4(0,2-1,1)$ & 0,07 & $0,9(0,3-2,9)$ & 0,98 & $0,9(0,3-2,7)$ & 0,84 \\
Dermatosis & $454(17,6 \%)$ & $1,5(1,1-1,9)$ & $<0,01$ & $1,2(0,8-1,8)$ & 0,31 & $1,1(0,7-1,7)$ & 0,67 \\
Posturas forzadas & $455(17,7 \%)$ & $1,3(1,0-1,8)$ & 0,03 & $1,8(1,2-2,7)$ & $<0,01$ & $1,8(1,2-2,7)$ & $<0,01$ \\
\hline
\end{tabular}

A pesar de que el ajuste por edad, sexo y estos y la ocupación diluyen la relación con los riesgos laborales, es interesante comprobar como una vez determinados, aún después de dicho ajuste, muestran una elevada tendencia a la asociación. El propio diseño del estudio es una limitación es este sentido; estudios con mayor potencia y otros diseños de carácter longitudinal pueden llegar a evidenciar asociaciones que, fisiopatológicamente, pueden ser pausibles, como p.e. en el caso del ruido, con quien Willich et al. ${ }^{2}$ encuentran valores superponibles a los obtenidos en este análisis, de forma significativa cuando la exposición es exclusivamente en horario nocturno.

Mención especial merece el análisis de las posturas forzadas y la significación numérica de su relación con el riesgo cardiovascular, que desaparece al ajustar por los factores relacionados con el mismo: el cálculo de la asociación mediante técnica de regresión logística (en los casos de elevada prevalencia del factor de estudio) tiende a sobreestimar el efecto, lo que, junto con la heterogénea distribución de los factores en los trabajadores expuestos a dicho riesgo podría explicar dicho resultado.

Una limitación del estudio viene determinada por la falta de consideración de riesgos psicosociales en las evaluaciones de riesgo, de modo que estos no han sido suficientemente considerados en el análisis del modelo. Del mismo modo que en las evaluaciones al uso se contemplan formalmente riesgos laborales clásicos y la gestión de los mismos, riesgos emergentes relacionados con la esfera psicosocial no están suficientemente recogidos ni debidamente evaluados. En este sentido, se han demostrado aumento de los factores de riesgo y de la mortalidad de causa cardiovascular en trabajadores industriales sometidos a estrés laboral ${ }^{8,12}$.

Los cambios en el mercado laboral propician la aparición de nuevos riesgos y la transformación de otros, a los que la salud laboral debe ser receptiva para proporcionar medidas de prevención eficaces $y$, en este sentido, es preciso evaluar convenientemente estos riesgos emergentes y considerarlos en nuevos estudios.

En lo referente a la prevalencia de los factores de RCV analizados, el comportamiento de la HTA, el colesterol plasmático o la diabetes, presentan valores muy similares a los de los estudios de características análogas efectuados en nuestro medio $5,6,7,10,11$. Sin embargo, es preciso destacar una disminución en el consumo de tabaco respecto a la mayoría de ellos, con una prevalencia de fumadores superiores en por- 
centaje hasta en 15 puntos. La entrada en vigor de la Ley antitabaco y su repercusión sobre la actividad laboral es una buena noticia en el control de los factores de riesgo cardiovascular y deberá medirse el impacto real de la implantación de la misma, tanto en términos económicos como de salud.

El estudio no es extrapolable a población general (por el conocido sesgo del trabajador sano cuando se utiliza población laboral) y, posiblemente, tampoco a población laboral del resto del estado, donde la distribución de los factores de RCV y la morbimortalidad a ellos asociada no siguen una distribución uniforme ${ }^{7}$.

La obtención de un porcentaje elevado de individuos con factores de riesgo y un riesgo global de tipo bajo-ligero debe atribuirse al uso de las tablas de RCV utilizadas, puesto que son más conservadoras, como se ha mencionado, en la determinación de dicho riesgo. Además, no debe obviarse la voluntariedad de los reconocimientos médicos actuales que puede actuar de diferentes formas según la interpretación que queramos realizar, ya sea favoreciendo o dificultando la asistencia en función del estado básico de salud.

\section{CONCLUSIONES}

Los factores de RCV no se asocian significativamente con ninguno de los riesgos laborales considerados. No obstante, su prevalencia es mayor en los sectores productivos de industria y construcción, y debe ser en estos donde los servicios de salud laboral deben priorizar sus actuaciones de prevención y promoción de la salud.

Los estilos de vida y las conductas saludables, reflejados en diferencias de los factores de RCV, difieren en los diferentes sectores de actividad. Además de priorizar las actuaciones en algunos de ellos, posiblemente deban elaborarse estrategias específicas en su abordaje, teniendo en cuenta las características propias de cada uno de ellos.
Como consideraciones finales, los servicios de salud laboral son un recurso excelente para la detección y el control de factores de riesgo cardiovascular, donde la prevención primaria y la promoción de la salud juegan un papel inestimable. El consejo dietético, modificaciones de conducta sobre el hábito del tabaco o la adopción de medidas higiénicas pueden suponer una importante disminución de las tasas de morbimortalidad derivada de patología cardiovascular ${ }^{9,13}$.

Las enfermedades cardiovasculares son actualmente un problema de salud pública de primera magnitud. El papel de los servicios de salud laboral no está suficientemente bien definido y en muchas ocasiones resulta insuficiente en el abordaje de estos problemas. Es preciso coordinar los recursos de los dispositivos asistenciales del sistema público de salud y los servicios de salud laboral para lograr el objetivo de una disminución eficaz de la morbimortalidad derivada de patología cardiovascular.

El autor declara la no existencia de conflicto de intereses.

\section{AGRADECIMIENTOS}

A la Dra. Cristina Abadia, Médico del Trabajo por su revisión al manuscrito inicial, sus siempre oportunos comentarios y, sobre todo, su amistad.

A la Dra. Paula Rivas, Médico del Trabajo por su colaboración en la búsqueda bibliográfica, sus comentarios durante el desarrollo del trabajo y su lectura crítica.

A M ${ }^{\mathrm{a}}$ Carmen Tamame, por su inestimable ayuda en la traducción al inglés.

A Sònia, Guillem i Xènia, per les estones que no us he pogut dedicar. Aquest cop si que anirem a veure en Mickey. 


\section{BIBLIOGRAFÍA}

1. Sánchez E. Estimació del risc cardiovascular a l'atenció primària. Document d'avaluació. Consulta técnica. Agencia d'avaluació de Tecnología i Recerca Mèdica. Barcelona 2003.

2. Willich SN, Wegscheider K, Stallmann M, Keil T. Noise burden and the risk of myocardial infarction. European Heart Journal (2006) 27, 276-282.

3. The European Heart Network. Social Factors, Work, Stress and Cardiovascular Disease Prevention in the European Union. EU 1998. www.ehnheart.org

4. Marrugat J, Solanas P, D'Agostino R, Sullivan L, Ordovas J, Cordón F, et al. Estimación del riesgo coronario en España mediante la ecuación de Framingham calibrada. Rev Esp Cardiol. 2003; 56: 253-61.

5. Castán FJ. Gutiérrez M. Factores de riesgo cardiovascular y tipo de actividad en una población laboral. Prevención, trabajo y salud 2004;29 : 18-43.

6. Sánchez MA, Román J, Calvo E, Gómez T, Fernández A, Sáinz JC, et al. Prevalencia de factores de riesgo vascular en la población laboral española. Rev Esp Cardiol 2006; 59: 421 - 430

7. Grupo de trabajo del INSHT. Factores de riesgo cardiovascular en la población laboral española. Prevención, Salud y Trabajo 2000:5:11-23
8. Kivimäki M, Leino-Arjas P., Luukkonen R., Riihimäki R, Vahtera J, Kirjonen J. Work stress and risk of cardiovascular mortality: prospective cohort study of industrial employees. BMJ 2002;325;857861.

9. Souza LA. Fernandes de Godoy M. Fatores de Risco Cardiovascular e Mortalidade. Seguimento em Longo Prazo (até 20 anos) em Programa Preventivo Realizado pela Medicina Ocupacional. Arquivos Brasileiros de Cardiologia 2005;85 (1): 20-25

10. Martínez-González MA, Hernández I., Zabala M.J, Alzugaray M, Duaso MJ, Ferrer A., Lameiro F. Prevalencia de los factores de riesgo cardiovascular en los trabajadores de una fábrica de Navarra. Anales del Sistema Sanitario de Navarra.1999 Disponible en: http://www.cfnavarra.es/ salud/anales/textos/vol22/n1/orig2.html

11. Grima A., Alegría E. Jover P. Prevalencia de los factores de riesgo cardiovascular clásicos en una población laboral mediterránea de 4.996 varones. Rev Esp Cardiol 1999; 52: 910-918.

12. Belkic K, Schnall P, Landsbergis P, Baker D. The workplace and cardiovascular health: conclusions and thoughts for a future agenda. Occup Med. 2000 Jan-Mar;15(1):307-21, v-vi.

13. Koffman D., Goetzel R., Anwuri V., Shore K., Orenstein D., LaPier T. Heart Healthy and Stroke Free Successful Business Strategies to Prevent Cardiovascular Disease. Am. J of Prev. Med.2005; (29):113-121. 